Yasser Sakr

\title{
Understanding the microcirculation in sepsis: still a long way to go...
}

Received: 31 March 2011

Accepted: 1 April 2011

Published online: 30 April 2011

(C) Copyright jointly held by Springer and ESICM 2011

This editorial refers to the article available at: doi:10.1007/s00134-011-2227-z.

\section{Y. Sakr (®)}

Department of Anesthesiology and Intensive Care, Friedrich Schiller University, Erlanger Allee 101, 07747 Jena, Germany

e-mail: Yasser.Sakr@med.uni-jena.de

Tel.: +49-3641-9323248

Fax: +49-3641-9323122
In this issue of Intensive Care Medicine, Dyson et al. [1] present the results of an experimental study in which they evaluated the temporal changes in myocardial function and regional tissue oxygenation in peripheral and deep organs in a rat model of fecal peritonitis. They observed an early decline in regional tissue oxygenation, as assessed by measurement of tissue partial pressure of oxygen $\left(\mathrm{tPO}_{2}\right)$, especially in animals with low stroke volume at baseline. A stepwise increase in $\mathrm{FiO}_{2}$ (oxygen challenge test) failed to increase the $\mathrm{tPO}_{2}$ sufficiently during the early phase of sepsis, but the response to oxygen challenge improved in all organ beds except the kidney by $24 \mathrm{~h}$. The strengths of this study [1] are that it provides information about cardiovascular function at both global and regional levels and that it evaluated regional perfusion simultaneously in several tissues-muscle, bladder, renal cortex, and liver. This multilevel approach would be expected to provide insight into the pathophysiology of sepsis and organ dysfunction.
Maintenance of adequate tissue oxygen transport is a keystone in the management of critically ill patients. This is conceptually achieved at three main levels: global, regional, and microcirculatory. Whereas global hemodynamics ultimately drives tissue perfusion, the microcirculation is the compartment in direct proximity to the cells and plays an essential role in the pathophysiology of sepsis [2]. Although changes in global hemodynamics have been reported to be discordant with microcirculatory alterations in sepsis [3], a critical decrease in global perfusion would nevertheless be expected to influence microvascular perfusion. In the study by Dyson et al. [1], animals were resuscitated according to a fixed fluid regime and not according to physiological targets. In the early phase of sepsis during which changes in $\mathrm{tPO}_{2}$ were observed, it is difficult to determine whether the alterations were because of microcirculatory failure or merely inadequate, low global oxygen delivery $\left(\mathrm{DO}_{2}\right)$. Indeed, the alterations in $\mathrm{tPO}_{2}$ were mainly observed in animals with low stroke volume, which raises concerns about the adequacy of volume resuscitation in this group. The differences in $\mathrm{tPO}_{2}$ between the early and the late phases of sepsis may, therefore, be explained by the presence of hypovolemia and resultant low $\mathrm{DO}_{2}$ during the early phase. This hypothesis is also supported by the fact that animals with higher stroke volume, described by the authors as having "good prognosis" sepsis, had preserved $\mathrm{tPO}_{2}$ in all the studied organs. Nevertheless, early microvascular alterations have been reported previously in experimental and clinical studies [2], so it is possible that the effects observed by Dyson et al. [1] were indeed the result of such changes.

Assessment of the microcirculation is not easy and should ideally be performed by direct evaluation of the structure of the microcirculation (i.e., functional capillary density, capillary perfusion, and heterogeneity of perfusion) using direct imaging techniques and evaluation of microcirculatory function in terms of the quality of microvascular perfusion (oxygenation) and reactivity [4]. 
Polarography is the method most frequently used to assess $\mathrm{tPO}_{2}$, but has several limitations, including possible tissue damage during insertion, reduced accuracy at $\mathrm{tPO}_{2}$ values less than $5 \mathrm{mmHg}$, effects on local $\mathrm{PO}_{2}$ as a result of oxygen consumption by the electrode, and that it is not suitable for repeated measurements [5]. Oxygen sensors based on optical fluorescence technology, such as those used in the study by Dyson et al., may have potential advantages over devices based on polarographic electrodes. These oxygen sensors are sensitive in the physiologically relevant $\mathrm{PO}_{2}$ range $(0-100 \mathrm{mmHg})$, do not consume oxygen, and can effectively provide real-time measurements because of their rapid response time $(5-10 \mathrm{~s})[6,7]$. The method is, however, temperature sensitive, so quantification requires temperature correction, which is accomplished by thermocouples attached to the fiber-optic probes. In addition, mechanical forces produced by the tip and shaft of needle electrodes, or the high pressure applied by surface electrodes, may cause tissue damage or induce local alterations in perfusion [8]. Importantly, oxygen electrodes do not directly assess the microcirculation, so they cannot evaluate the heterogeneity of microvascular perfusion and microvascular shunting in sepsis [9]. Adjacent areas of diminished and increased microvascular perfusion in the catchment area of these sensors may thus falsely be reported as normal or high $\mathrm{tPO}_{2}$ values.

Although $\mathrm{tPO}_{2}$ may reflect the balance between oxygen supply and demand as Dyson et al. [1] allude to, quantification of this relationship can be troublesome. The increase in $\mathrm{tPO}_{2}$ during an oxygen challenge test does not necessarily mean that microvascular perfusion has improved. Increased $\mathrm{PO}_{2}$ in previously patent microvascular units may increase the measured $\mathrm{tPO}_{2}$ values because of the high affinity of these sensors to oxygen, without any recruitment of new microvascular units in the catchment area of the electrodes. In addition, in the presence of microvascular shunting, the $\mathrm{tPO}_{2}$ response to an oxygen challenge test may be normal despite the fact that oxygen is not available for direct exchange with the cells. In this case, $\mathrm{tPO}_{2}$ does not reflect the balance between oxygen supply and demand.

Other points that should be taken into consideration in the interpretation of the data provided by Dyson et al. [1] relate to the experimental design. Although the animal model used is relatively stable, the study does not provide real temporal changes, because measurements in the early and late phases were not performed in the same animal. It is possible that the animals included in the late sepsis group may have had a different hemodynamic and tissue oxygenation profile from those investigated in the earlier phase. Indeed, a considerable number of animals died before reaching this point, which suggests that the included animals were those that did well. In addition, the study groups were not classified according to survival but according to stroke volume at the beginning of the experiment. In the presence of low end-diastolic volume, low stroke volume may be indicative of hypovolemia and not of myocardial dysfunction. This notion is supported by the fact that both end-diastolic volume and stroke volume increased considerably after fluid loading in this study.

The study by Dyson et al. [1] is a useful addition to the literature in this field of research, providing one more piece of the puzzle as we try to understand more fully the patterns of tissue oxygenation in sepsis and their relation to outcome. Nevertheless, the data should be interpreted with some caution, bearing in mind the methodological limitations.

\section{References}

1. Dyson A, Rudiger A, Singer M (2011) Temporal changes in tissue cardiorespiratory function during faecal peritonitis. Intensive Care Med. doi: 10.1007/s00134-011-2227-z

2. Ince $C$ (2005) The microcirculation is the motor of sepsis. Crit Care 9(Suppl 4):S13-S19

3. Sakr Y, Dubois MJ, De Backer D, Creteur J, Vincent JL (2004) Persistent microcirculatory alterations are associated with organ failure and death in patients with septic shock. Crit Care Med 32:1825-1831

4. Sakr Y (2010) Techniques to assess tissue oxygenation in the clinical setting. Transfus Apher Sci 43:79-94
5. Swartz HM (2002) Measuring real levels of oxygen in vivo: opportunities and challenges. Biochem Soc Trans 30:248-252

6. Dunn JF, Nwaigwe CI, Roche M (1999) Measurement of arterial, venous, and interstitial pO2 during acute hypoxia in rat brain using a time-resolved luminescence-based oxygen sensor. Adv Exp Med Biol 471:43-48
7. Collingridge DR, Young WK, Vojnovic B, Wardman P, Lynch EM, Hill SA, Chaplin DJ (1997) Measurement of tumor oxygenation: a comparison between polarographic needle electrodes and a time-resolved luminescence-based optical sensor. Radiat Res 147:329-334

8. Hopf HW, Hunt TK (1994) Comparison of Clark electrode and optode for measurement of tissue oxygen tension. Adv Exp Med Biol 345:841-847

9. De Backer D, Dubois MJ (2001) Assessment of the microcirculatory flow in patients in the intensive care unit. Curr Opin Crit Care 7:200-203 